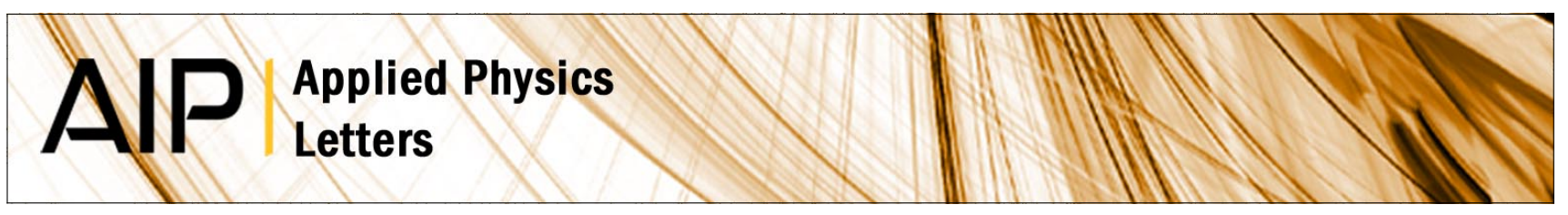

\title{
Subnanosecond ellipticity detector for laser radiation
}

S. D. Ganichev, J. Kiermaier, W. Weber, S. N. Danilov, D. Schuh et al.

Citation: Appl. Phys. Lett. 91, 091101 (2007); doi: 10.1063/1.2775809

View online: http://dx.doi.org/10.1063/1.2775809

View Table of Contents: http://apl.aip.org/resource/1/APPLAB/v91/i9

Published by the American Institute of Physics.

\section{Related Articles}

$\mathrm{NbTiN}$ superconducting nanowire detectors for visible and telecom wavelengths single photon counting on Si3N4 photonic circuits

Appl. Phys. Lett. 102, 051101 (2013)

Superconducting single-photon counting system for optical experiments requiring time-resolution in the picosecond range

Rev. Sci. Instrum. 83, 123103 (2012)

Enhanced performance of resonant sub-terahertz detection in a plasmonic cavity

Appl. Phys. Lett. 100, 083506 (2012)

Plasmonic detector/spectrometer of subterahertz radiation based on two-dimensional electron system with embedded defect

Appl. Phys. Lett. 100, 082102 (2012)

Ultra-low-noise MoCu transition edge sensors for space applications

J. Appl. Phys. 109, 084507 (2011)

\section{Additional information on Appl. Phys. Lett.}

Journal Homepage: http://apl.aip.org/

Journal Information: http://apl.aip.org/about/about_the_journal

Top downloads: http://apl.aip.org/features/most_downloaded

Information for Authors: http://apl.aip.org/authors

\section{ADVERTISEMENT}

\section{AIP Applied Physics Letters}

\section{EXPLORE WHAT'S NEW IN APL}

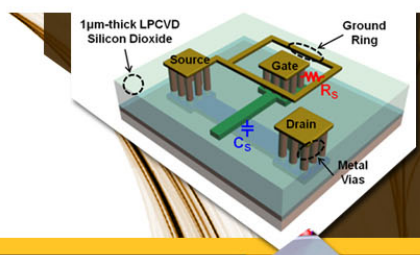

SURFACES AND INTERFACES

Focusing on physical, chemical, biological, structural, optical, magnetic and electrical properties of surfaces and interfaces, and more.. 


\title{
Subnanosecond ellipticity detector for laser radiation
}

\author{
S. D. Ganichev, ${ }^{\text {a) }}$ J. Kiermaier, W. Weber, S. N. Danilov, D. Schuh, Ch. Gerl, \\ W. Wegscheider, and W. Prettl \\ Terahertz Center, University of Regensburg, D-93040, Regensburg, Germany \\ D. Bougeard and G. Abstreiter \\ Walter-Schottky Institute, Technical University of Munich, D-85748 Garching, Germany
}

(Received 9 July 2007; accepted 2 August 2007; published online 27 August 2007)

\begin{abstract}
A room temperature detector of terahertz laser radiation ellipticity has been developed based on the simultaneous measurements of three different photoelectric phenomena: circular photogalvanic effect, linear photogalvanic effect, and photon drag effect. Each of these effects, which have subnanosecond time constants, is monitored by different detector units stacked together in one detector. (C) 2007 American Institute of Physics. [DOI: 10.1063/1.2775809]
\end{abstract}

The detection of the polarization state, in particular, the ellipticity of transmitted, reflected, or scattered light represents a powerful technique for analyzing the optical anisotropy of various media such as plasmas, gases, solids and solid surfaces, as well as biological tissues. Illustrative examples are tokamak polarimetry to measure electric and magnetic fields in ionized gases as well as the plasma density, monitoring and control of material preparation and processing, circular dichroism spectroscopy and optical rotary dispersion of proteins and molecules, contactless and nondestructive measurements of surfaces and very thin films, and analysis of gas and aerosol constituents in the Earth's atmosphere by polarization sensitive optical remote sensing. The established way to gain information about the polarization state is the use of optical elements, which allow the determination of the optical path difference. Some tasks need measurement of dynamic ellipticity of polarized light. This time resolved measurement of ellipticity is so far realized by transmitting elliptical light through a rapidly rotating analyzing prism in front of a photodetector or by using of a pumpprobe method.

Here, we report on a pure photoelectrical detector system operating at room temperature based on the simultaneous measurement of photocurrents obtained from three unbiased semiconductor detector elements in response to laser radiation. The first element provides information about the radiation helicity describing the rotation of the light's polarization vector by applying the circular photogalvanic effect (CPGE) in quantum wells (QWs). ${ }^{1}$ In the second, the signal of the linear photogalvanic effect ${ }^{1}$ (LPGE) in a QW made of another material allows the determination of the azimuth angle. The third detector element is a photon drag detector made of bulk germanium. ${ }^{1}$ It provides a polarization independent signal which is used to measure the intensity of radiation, which is needed to calibrate the whole system. While the principle of ellipticity detection can be applied in a wide wavelength range, including visible, in this letter we present the detector for terahertz laser radiation.

The ellipticity detector is sketched in Fig. 1. The first (U1) and the second (U2) detector elements are made of GaAs QW and SiGe QW structures, respectively, both grown by molecular beam epitaxy on (113)-oriented substrates in

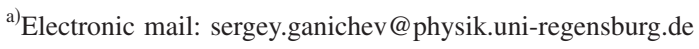

order to provide photogalvanic response at normal incidence of radiation on the samples. ${ }^{1}$ In the terahertz range these detector units are practically transparent and therefore, the units can be mounted one behind the other for illumination with the same laser beam. The U1 element is a molecular beam epitaxy (MBE) grown $p$-GaAs $/ \mathrm{Al}_{0.3} \mathrm{GaAs}_{0.7}$ multiple QW structure containing 20 wells of $10 \mathrm{~nm}$ width with free hole densities of about $2 \times 10^{11} \mathrm{~cm}^{-2}$ per QW. The U2 element is a MBE grown $\mathrm{Si} / \mathrm{Si}_{0.75} \mathrm{Ge}_{0.25} / \mathrm{Si}$ single $\mathrm{QW}$ of $5 \mathrm{~nm}$ width. The QW structure is one-side boron doped with a free carrier density in the well of about $8 \times 10^{11} \mathrm{~cm}^{-2}$. For both square shaped structures of $5 \times 5 \mathrm{~mm}^{2}$ size, a pair of Ohmic contacts is centered on opposite sample edges along the [1̄o] crystallographic axis (see Fig. 1). As last element (U3) we use a photon drag detector. It consists of a Ge:Sb cylinder of $5 \mathrm{~mm}$ diameter about $30 \mathrm{~mm}$ long with plane parallel end faces and ring shaped electric contacts at both ends. The doping level is about $10^{14} \mathrm{~cm}^{-3}$. The signal voltages are picked up independently from each detector unit in a closed circuit configuration across a $50 \Omega$ load resistor. Signals are fed into an amplifier with $20 \mathrm{~dB}$ amplification and a rise time of about $5 \mathrm{~ns}$ or directly measured by a digital oscilloscope.

The functionality of the ellipticity detector, its sensitivity, and time resolution are demonstrated using a pulsed terahertz $\mathrm{NH}_{3}$ laser ${ }^{1}$ which provides $100 \mathrm{~ns}$ pulses with a radiation power $P$ of about $100 \mathrm{~kW}$. Several lines of the $\mathrm{NH}_{3}$ laser between $\lambda=76$ and $280 \mu \mathrm{m}$ have been applied. We also use a cw-methanol laser in order to check the detector applicability for detection of the $118.8 \mu \mathrm{m}$ laser line important for tokamak applications (see, e.g., Ref. 2). Elliptically po-

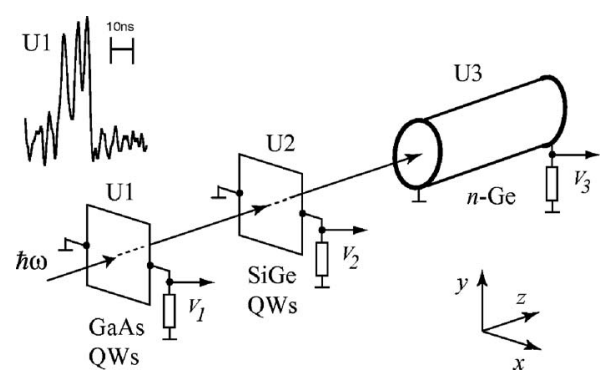

FIG. 1. Design of the detector of radiation ellipticity. The inset shows an oscilloscope trace of a signal pulse of $\lambda=148 \mu \mathrm{m}$ obtained by the detector unit U1. 


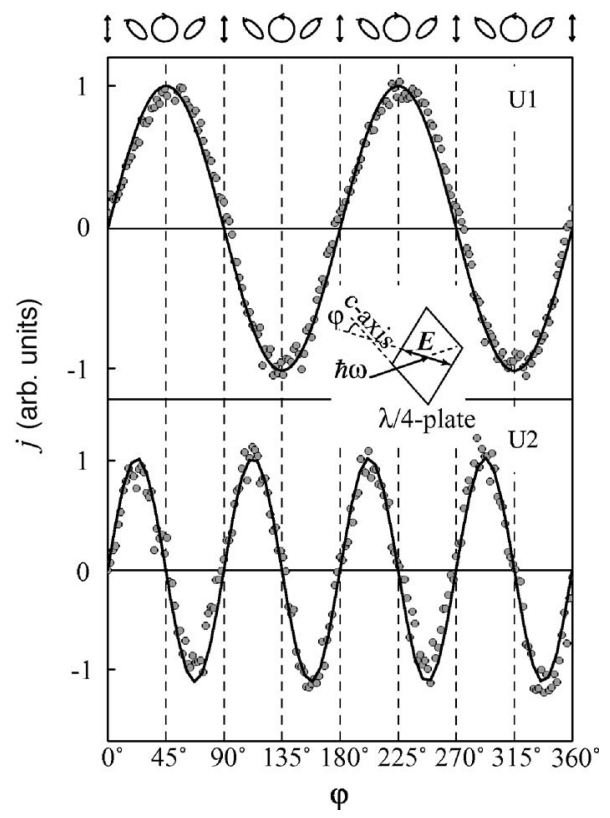

FIG. 2. Photoresponse of the GaAs (U1, upper panel) and SiGe (U2, lower panel) QWs as a function of the phase angle $\varphi$ defined in the inset. The voltage response is measured in both structures along the [1ㅣㅣ direction and normalized to the peak signal of each unit. Measurements are presented for $T=300 \mathrm{~K}$ and normal incidence of radiation at $\lambda=148 \mu \mathrm{m}$. The change of the azimuth, the shape of the ellipse, and the direction of rotation of the electric field vector by varying of $\varphi$ are sketched above. This variation of light ellipticity is obtained by rotation of the $\lambda / 4$ plate with respect to the polarization plane of laser radiation (see inset). Full lines are fits by CPGE (upper panel) and LPGE (lower panel) after Eq. (1). Fits are obtained using only ordinate scaling parameters.

larized light is generated by passing plane polarized light at normal incidence through a birefringent $\lambda / 4$ plate made of crystalline quartz. In order to vary the light ellipticity, we rotated the $\lambda / 4$ plate with respect to the polarization plane of the laser radiation by the angle $\varphi$ between the optical axis of the $\lambda / 4$-plate and the direction of the initial polarization. In that way we obtain by varying $\varphi$ a change of the azimuth, the shape of the ellipse, and the direction of rotation of the electric field vector (see Fig. 2). The latter determines the radiation helicity $P_{\text {circ }}=\sin 2 \varphi$ given by $P_{\text {circ }}$ $=\left(\left|E_{\sigma+}\right|^{2}-\left|E_{\sigma-}\right|^{2}\right) /\left(\left|E_{\sigma+}\right|^{2}+\left|E_{\sigma-}\right|^{2}\right)$, where $\left|E_{\sigma+}\right|$ and $\left|E_{\sigma-}\right|$ are the amplitudes of right and left handed circularly polarized radiation, respectively.

In response to irradiation at normal incidence of the helicity sensitive detector U1, we obtained a voltage signal which resolves the temporal structure of the laser pulse intensity, as shown in the inset of Fig. 1. Figure 2 shows that the signal closely follows the radiation helicity which results in a $\sin 2 \varphi$ dependence. A signal, also resolving the laser pulse, has been detected by the second detector unit $\mathrm{U} 2$ made of the SiGe QW structure. In contrast to U1, in this case the signal varies as a $\sin 4 \varphi$ and vanishes for circularly polarized radiation. These angle dependences follow from the phenomenological theory of the photogalvanic effect. For irradiation at normal incidence of $\mathrm{C}_{s}$ symmetry structures, like in the present (113)-grown QWs, it yields

$$
\begin{aligned}
j_{x} & =\chi_{x x y}\left(E_{x} E_{y} *+E_{y} E_{x} *\right)+\gamma_{x z} \hat{e}_{z} E^{2} P_{\text {circ }} \\
& =\chi_{x x y} \hat{e}_{z} E^{2} \sin 4 \varphi+\gamma_{x z} \hat{e}_{z} E^{2} \sin 2 \varphi,
\end{aligned}
$$

where $x\|[1 \overline{1} 0], y\|[33 \overline{2}], \mathbf{E}$ is the complex amplitude of the electric field of the electromagnetic wave with the electric field amplitude $E_{0}=|\mathbf{E}|$ and the unit vector $\hat{e}$ points in the direction of light propagation. The photocurrent given by the tensor $\chi$ corresponds to the LPGE which arises due to an asymmetry of the scattering of free carriers by, e.g., phonons or static defects. The CPGE described by the pseudotensor $\gamma$ is caused by the transfer of the angular momentum of circularly polarized photons into a directed motion of free carriers. The angular dependences, $\sin 2 \varphi$ in the element U1 and $\sin 4 \varphi$ in the element $\mathrm{U} 2$, observed in the experiment, demonstrate that the photocurrent is caused predominately by CPGE in our GaAs QWs and by LPGE in the present SiGe structures. The signal obtained by the photon drag detector unit $\mathrm{U} 3$ is independent of the radiation ellipticity and is used for monitoring radiation power.

From Fig. 2, it follows that simultaneous measurements of all three signals allows the unambiguous determination of the radiation ellipticity. Indeed, a pair of signals of U1 and $\mathrm{U} 2$ obtained at an angle $\varphi$ is unique and this pair of voltages will never be repeated for variation of $\varphi$ between 0 - and $\pi$. The ratio of the signals of $\mathrm{U} 1$ and $\mathrm{U} 2$, and even the sign of this ratio, is different for different angles $\varphi$. By that the angular position of the ellipse (azimuth) can be derived from the strength of the LPGE (element U2) and its ellipticity from the helicity dependent CPGE (element U1). Due to rapid free carrier momentum relaxation at room temperature the response time of each detector unit is in the order of picoseconds. The practically achievable time resolution, however, is $R C$ limited by the design of the electric circuitry and by the bandwidth of cables and amplifiers. In the investigated wavelength range from 76 to $280 \mu \mathrm{m}$, we obtained that the voltage response of the ellipticity detector depends linearly on the power up to the strongest applied intensities of about $2 \mathrm{MW} / \mathrm{cm}^{2}$. Sensitivities of the detector units U1, $\mathrm{U} 2$, and $\mathrm{U} 3$ at the wavelength of $148 \mu \mathrm{m}$ are $3.2 \mathrm{mV} / \mathrm{kW}$ $\left(\varphi=45^{\circ}\right), 1.2 \mathrm{mV} / \mathrm{kW}\left(\varphi=22.5^{\circ}\right)$, and $35 \mathrm{mV} / \mathrm{kW}$, respectively. These values are obtained for $50 \Omega$ load resistance and $20 \mathrm{~dB}$ amplifier for the angles $\varphi$ corresponding to the maximum of the signal. The values of sensitivities are sufficient to detect short terahertz pulses of sources such as optically pumped molecular lasers and free-electron lasers. The method has also been applied to monitor the ellipticity of $\mathrm{cw}$ terahertz laser radiation by reducing the bandwidth of detection.

Finally, we would like to point out that the sensitivity of the described detector can be essentially improved by using a larger number of QWs. Further increase of sensitivity can be obtained by applying narrow band materials such as $\mathrm{HgTe}$ QWs in which most recently we observed CPGE signals more than an order of magnitude larger than that of GaAs QWs used here ${ }^{3}$ or in specially designed lateral structured QWs with enhanced asymmetry. Sensitive terahertz detectors of the type shown here, besides other applications, can be of particular interest for the control of current density profiles which is important for tokamak operation. ${ }^{4-8}$ We also note that the CPGE and LPGE are also observed at valence-toconduction band transitions, ${ }^{9-11}$ at direct intersubband transitions, ${ }^{1}$ and in wide band GaN semiconductor heterojunctions. ${ }^{12}$ Thus, the applicability of the CPGE/LPGE detection scheme may well be extended into the visible, the near-infrared, and in the midinfrared spectral ranges. 
501/6, Collaborative Research Center (SFB689), and Project SPP 1285.

${ }^{1}$ S. D. Ganichev and W. Prettl, Intense Terahertz Excitation of Semiconductors (Oxford University Press, Oxford, 2006), pp. 5 and 291.

${ }^{2}$ A. J. Donné, M. F. Graswinckel, M. Cavinato, L. Giudotti, E. Zilli, C. Gil, H. R. Koslowski, P. McCarthy, C. Nyhan, S. Prunty, M. Spillane, and C. Walker, Rev. Sci. Instrum. 75, 4694 (2004).

${ }^{3}$ B. Wittmann, S. N. Danilov, Z. D. Kvon, N. N. Mikhailov, S. A. Dvoretsky, R. Ravash, W. Prettl, and S. D. Ganichev, in IEEE Proceedings of the Joint 32nd International Conference on Infrared and Millimetre Waves and 15th International Conference on THz Electronics, UK, 2007 (unpublished), Vol. 2, pp. 773-774.

${ }^{4}$ S. A. Kazantsev, N. Ya. Polynovskaya, L. N. Pyatnitskii, and S. A. Edel'man, Sov. Phys. Usp. 31, 785 (1988).
${ }^{5}$ V. P. Gavrilenko, Measurement Techniques 35, 1315 (2005).

${ }^{6}$ F. DeMarco and S. E. Segre, Plasma Phys. 14, 245 (1972).

${ }^{7}$ A. Boileau, M. von Hellerman, W. Mandl, H. P. Summers, H. Weisen, and A. Zinoviev, J. Phys. B 22, L145 (1989).

${ }^{8}$ F. M. Levinton, R. J. Fonck, G. M. Gammel, R. Kaita, H. W. Kugel, E. T. Powell, and D. W. Roberts, Phys. Rev. Lett. 63, 2060 (1989).

${ }^{9}$ V. V. Bel'kov, S. D. Ganichev, Petra Schneider, C. Back, M. Oestreich, J. Rudolph, D. Hägele, L. E. Golub, W. Wegscheider, and W. Prettl, Solid State Commun. 128, 283 (2003).

${ }^{10}$ C. L. Yang, H. T. He, Lu Ding, L. J. Cui, Y. P. Zeng, J. N. Wang, and W. K. Ge, Phys. Rev. Lett. 96, 186605 (2006).

${ }^{11}$ M. Bieler, N. Laman, H. M. van Driel, and A. L. Smirl, Appl. Phys. Lett. 86, 061102 (2005).

${ }^{12}$ W. Weber, S. D. Ganichev, S. N. Danilov, D. Weiss, W. Prettl, Z. D. Kvon, V. V. Bel'kov, L. E. Golub, Hyun-Ick Cho, and Jung-Hee Lee, Appl. Phys. Lett. 87, 262106 (2005). 